\title{
Capsule depolymerase overexpression reduces Bacillus anthracis virulence
}

Correspondence

Angelo Scorpio

scorpioa@nbacc.net

Arthur Friedlander

arthur.friedlander@us.army.mil

Received 26 October 2009

Revised 4 January 2010

Accepted 27 January 2010

\author{
Angelo Scorpio,† Donald J. Chabot, William A. Day,‡ Timothy A. Hoover \\ and Arthur M. Friedlander
}

United States Army Medical Research Institute of Infectious Diseases, Frederick, MD 21702, USA

\section{INTRODUCTION}

Bacillus anthracis is the causative agent of anthrax and is considered one of the most important biological warfare threats. The virulence of $B$. anthracis is primarily attributed to genes located on pathogenicity islands of pX01 and pX02. A tripartite toxin composed of protective antigen, lethal factor and oedema factor is encoded on pX01, while genes for the synthesis of an antiphagocytic, poly- $\gamma$-Dglutamic acid capsule are carried on pX02 (Mock \& Fouet, 2001). The capsule operon is composed of five genes, cap $B, C, A, D$ and $E$. Capsule depolymerase, encoded by capD, is a $\gamma$-glutamyl transpeptidase (GGT)-family protein that autocatalytically forms a heterodimer consisting of $35 \mathrm{kDa}$ and $15 \mathrm{kDa}$ subunits. CapD shares $32 \%$ identity with the Bacillus subtilis GGT and $35 \%$ identity with a recently reported Francisella CapD homologue (Su et al., 2007). Despite this amino acid sequence similarity, the B. subtilis GGT is reported to be secreted (Kimura et al., 2004), while B. anthracis CapD is membrane associated (Candela \& Fouet, 2005). The individual subunits of CapD associate to form a functional enzyme that cleaves the $\gamma$-linked peptide bond of the polyglutamate capsule,

†Present address: Battelle National Biodefense Institute, Frederick, MD 21702, USA.

‡Present address: Ventana Medical Systems, Tucson, AZ 85755, USA.

Abbreviation: GGT $\gamma$-glutamyl transpeptidase. transferring glutamate to a water molecule (hydrolysis) or an amino acid (transpeptidation). This mechanism results in release of low-molecular-mass capsule and attachment of the capsule to the peptidoglycan layer (Candela \& Fouet, 2005; Makino et al., 2002; Uchida et al., 1993), specifically, to the side-chain amino group of mesodiaminopimelic acid (Richter et al., 2009). We previously demonstrated that the capsule-degrading activity of recombinant CapD can be exploited to enzymically remove the capsule from the surface of bacilli, resulting in enhanced phagocytic killing by human neutrophils and protection in mouse models of anthrax infection (Scorpio et al., 2007, 2008).

We observed by proteomic analysis that CapD, like CapB, $\mathrm{C}$ and $\mathrm{A}$, is associated with the membrane during growth in laboratory media and in vivo (Scorpio et al., 2005). An interesting finding from this study was that while CapB, C and $\mathrm{A}$ were detected in similar amounts, CapD was only weakly detected in whole-cell and membrane-enriched preparations from laboratory-grown bacilli and was detected in amounts $<1 \%$ that of CapB, C and $\mathrm{A}$ in bacilli isolated from infected guinea pigs. Nevertheless, a functional CapD has been shown to be required for virulence in mice and guinea pigs (Candela \& Fouet, 2005; Makino et al., 2002; Richter et al., 2009). These observations led us to hypothesize that increased expression of capD may result in more capsule being released from the 
surface of bacilli, potentially allowing the bacilli to be more readily phagocytosed. To investigate this, in the present study we upregulated capD expression using transcriptional fusions to various promoters and modifications to translation signals. Strains with higher capD expression had less surface-associated capsule, increased susceptibility to phagocytic killing, and reduced virulence in guinea pigs compared with strains with wild-type levels of capD expression. We propose that $B$. anthracis may have evolved weak $c a p D$ expression signals resulting in wildtype amounts of cell-associated capsule and a ratio of low- to high-molecular-mass capsule that is optimal for virulence.

\section{METHODS}

Bacterial strains and animals. Strains and plasmids used in this study are listed in Table 1. B. anthracis Ames (pX01 $\left.{ }^{+} \mathrm{pX02}{ }^{+}\right)$and $\triangle \mathrm{ANR}\left(\mathrm{pX01}{ }^{-} \mathrm{pX02}^{-}\right)$were cultured in brain heart infusion (BHI) broth with $0.8 \%$ sodium bicarbonate and $5 \%$ carbon dioxide or on NBY bicarbonate agar plates at $37{ }^{\circ} \mathrm{C}$ with $5 \%$ carbon dioxide (Chabot et al., 2004). Escherichia coli strains DH5 $\alpha$ and GM2163 were used for cloning and strain construction. Antibiotics were used at the following concentrations: kanamycin, $50 \mu \mathrm{g} \mathrm{ml}^{-1}$ for E. coli strains and $20 \mu \mathrm{g} \mathrm{ml}^{-1}$ for B. anthracis; and spectinomycin, $200 \mu \mathrm{g} \mathrm{ml}^{-1}$ for B. anthracis. B. anthracis spores were prepared as previously described (Chabot et al., 2004). Female Hartley guinea pigs, 350-400 g, were obtained from Charles River Laboratories. Female Swiss Webster mice, 6-8 weeks old, were obtained from the National Cancer Institute.

Construction of a capD knockout strain of B. anthracis Ames. All but the first 180 and last 105 bases of capD were deleted by homologous recombination with plasmid pE03 (Mendelson et al., 2004) containing the promoterless aphA-3 cassette from pUC18k (Menard et al., 1993) flanked by regions upstream and downstream of capD. The upstream flank was made by PCR with a $5^{\prime}$ NotI site using forward primer $5^{\prime}$-ATAAGAATGCGGCCGCGAAGCTGATCTTGA-
CTATGTGGGTGCTGGTG-3' and reverse primer 5'-TCCCCCGGGTCAACCGCAAGGGGGTGAGAGGCACTCACTC- ${ }^{\prime}$. The downstream flank was made by PCR with a $3^{\prime} K p n I$ site with forward primer 5' ${ }^{\prime}$-TCCCCCCGGGGCAGAAGAAATGGAACTTGGAAATCAAATAAATAGGAGG-3' and reverse primer $5^{\prime}$-GGGGTACCCCAGTAATTAAGACACCGTCAAATCCGT TCTAC-3'.

Flanks were cut with the appropriate enzyme plus XmaI and ligated simultaneously into pE03 after digestion with KpnI and NotI. The aphA-3 nonpolar mutagenesis cassette from pUC18k was then cloned into the XmaI site. Selection for homologous recombination was performed as described previously (Mendelson et al., 2004). Stable knockouts were selected for by loss of erythromycin resistance $(5 \mu \mathrm{g}$ $\mathrm{ml}^{-1}$ in LB broth) and were confirmed by PCR with internal and external primers. Colonies appeared hypermucoid, compared to wildtype, when grown on NBY bicarbonate plates at $37{ }^{\circ} \mathrm{C}$ with $5 \% \mathrm{CO}_{2}$.

Construction of capD fusion strains. Transcriptional fusions of capD were created by cloning an approximately $300 \mathrm{bp}$ PCR product encoding the 3' end of hsp60 (BA0267), EFTu (BA0108) or capA into the shuttle plasmid pASD2 (Day et al., 2007) as an XmaI-XhoI fragment. The ORF of capD including $29 \mathrm{bp}$ upstream from the putative start codon was cloned $3^{\prime}$ to the above fragments as an XhoI$K p n I$ fragment. The reverse primer used to generate capD for fusion to capA was designed to anneal at the $3^{\prime}$ end of capE. Additional PCR products were generated that included the $c a p D O R F$ and contained a consensus ribosome-binding site (RBS), AGGAGGT, and a TTG start codon with the forward primer 5'-GCGGCGCTCGAGAGGAGGTCAACAACTTGAATTCCTTTAAATGGGGAAAGA-3' and the above $c a p D$ reverse primers. Additional $c a p D$ ORFs were generated that contained a consensus RBS and an ATG start codon or the wild-type RBS and a TTG start codon. Primers employed to generate the amplicons are listed in Table 2. The pASD2 plasmid constructs were passaged through E. coli GM2163 before electroporation into B. anthracis strains. B. anthracis $\triangle \mathrm{ANR}\left(\mathrm{pX01}{ }^{-} \mathrm{pX02^{- } )}\right.$ was transformed with the plasmids containing capD fused to $h s p 60$ or $E F T u$, and kanamycin/spectinomycin-resistant colonies were isolated on Luria-Bertani (LB) agar containing antibiotics. The plasmids were integrated at the $3^{\prime}$ end of $h s p 60$ or EFTu as previously described (Day et al., 2007) and the linkages transferred to fully virulent B. anthracis Ames by CP-51 phage transduction (Green et al., 1985). This resulted

Table 1. Bacterial strains and plasmids used in this study

\begin{tabular}{|c|c|c|}
\hline Strain or plasmid & Relevant characteristics & Source \\
\hline \multicolumn{3}{|l|}{ B. anthracis } \\
\hline Ames & Wild-type & Lab collection \\
\hline$\triangle \mathrm{ANR}$ & $\mathrm{pXO}^{-} \mathrm{pXO}^{-}$ & Lab collection \\
\hline \multicolumn{3}{|l|}{ E. coli } \\
\hline DH5 $\alpha$ & & New England Biolabs \\
\hline GM2163 & $\mathrm{dam} \mathrm{dcm}$ & New England Biolabs \\
\hline \multicolumn{3}{|l|}{ Plasmids } \\
\hline pE03 & Integrative plasmid & Mendelson et al. (2004) \\
\hline pASD2 & Integrative plasmid & Day et al. (2007) \\
\hline pASD2hsp60capD & Fusion of the $3^{\prime}$ end of $h s p 60$ to capD ORF & This work \\
\hline pASD2hsp60capDRBS & Fusion of the $3^{\prime}$ end of $h s p 60$ to $c a p D$ ORF with engineered RBS for capD & This work \\
\hline pASD2EFTucapDRBS & Fusion of the $3^{\prime}$ end of EFTu to capD ORF with engineered RBS for capD & This work \\
\hline pASD2capAcapDRBSATG & Fusion of the $3^{\prime}$ end of $c a p A$ to $c a p D$ ORF with engineered RBS and ATG capD start codon & This work \\
\hline pASD2capAcapDTTG & Fusion of the $3^{\prime}$ end of capA to capD ORF with engineered TTG capD start codon & This work \\
\hline pASD2capAcapDRBSTTG & Fusion of the $3^{\prime}$ end of $c a p A$ to $c a p D$ ORF with engineered RBS and TTG start codon & This work \\
\hline pASD2capAcapDATG & Fusion of the $3^{\prime}$ end of capA to capD ORF with engineered ATG capD start codon & This work \\
\hline pASD2capAcapD & Fusion of the $3^{\prime}$ end of capA to capD ORF & This work \\
\hline
\end{tabular}


Table 2. Oligonucleotides used to create amplicons for construction of $c a p D$ fusion strains

F, Forward primer; R, reverse primer.

\begin{tabular}{|ll|}
\hline PCR product & \multicolumn{1}{c|}{ Primer sequences } \\
\hline \multirow{2}{*}{ hsp60 $3^{\prime}$ end } & F: 5'-GCGGCGCCCGGGTAGAAGAGCCAGTTC GTCAAATCGCAATCA \\
& R: 5'-GCGGCGCTCGAGTTACAT CATTCCGCCCATACCGCCCATGCC \\
EFTu $3^{\prime}$ end & F: 5'-GCGGCGCCCGGGCGTATTATCTAAAGAAGAAGGTGGACG \\
& R: 5'-GCGGCGCTCGAGTTACTCAACGATAGTAGCAACTACACC \\
capA 3' end & F: 5'-GCGGCGCCCGGGAGTGCACTTGTGCAATATCATTTACGTGAT \\
& R: 5' -GCGGCGCTCGAGTCAAGTTGTTGTCTCCACTGATACTTG ATT \\
& FapD ORF 5 -GCGGCGCTCGAGGAAAATCAAGTATCAGTG GAGACAACAACTTGA \\
& R: 5'-GCGGCGGGTACCCTATTT ATTTGATTTCCAAGTTCCATT \\
& R: 5'-GCGGCGGGTACCTTAGGGGTTAGCCTGTAGATAATCACTAAT* \\
\hline
\end{tabular}

${ }^{\star}$ Reverse primer used to generate capD ORF for fusion to capA.

in two copies of capD in the $h s p 60$ and EFTu fusions, one on the chromosome and one on pX02. The fusions of capD to capA were made by integrating the fusion plasmids at the $3^{\prime}$ end of cap $A$ in the B. anthracis Ames capD mutant. Genetic linkages were confirmed by dye terminator Sanger sequencing.

Analysis of CapD and capsule expression. Strains overexpressing capD and wild-type Ames were grown in BHI/bicarbonate broth at $37^{\circ} \mathrm{C}$ with shaking. Bacteria were collected by centrifugation and resuspended in PBS, $\mathrm{pH}$ 7.4. The cells were disrupted by sonication and the resulting lysates added to an equal volume of $2 \times$ SDS protein sample buffer. Samples were heated at $100{ }^{\circ} \mathrm{C}$ for $10 \mathrm{~min}$ and centrifuged for $2 \mathrm{~min}$. The extracted proteins were separated by SDSPAGE and stained with Gel Code Blue (Pierce Biotechnology). Expression of $c a p D$ was examined by immunoblotting (Invitrogen) and probed with mouse polyclonal anti-CapD antiserum at $1: 1000$ dilution. Capsule in culture supernatants from capD-overexpressing strains was visualized by electrophoresis in $1 \%$ agarose gels followed by staining with $0.3 \%$ methylene blue in $20 \%$ ethanol and destaining with water. In one experiment $9 \mu \mathrm{l}$ of the culture supernatant from EFTucapDRBS containing low-molecular-mass capsule was treated with $1 \mu \mathrm{l}(0.3 \mu \mathrm{g})$ recombinant $\mathrm{CapD}$ (prepared as described previously: Scorpio et al., 2007) and $1.1 \mu \mathrm{l} 1 \mathrm{M}$ serine. Mixtures with and without CapD were incubated at $37{ }^{\circ} \mathrm{C}$ for $2 \mathrm{~h}$. Capsule degradation by CapD was visualized by electrophoresis in $2 \%$ agarose gels followed by staining with methylene blue, as described above.

Neutrophil killing and macrophage adhesion assays. Neutrophils were isolated and killing assays performed as previously described (Scorpio et al., 2007). Briefly, heat-shocked spores were germinated $\left(2 \times 10^{7} \mathrm{ml}^{-1}\right)$ in BHI containing $0.8 \%$ sodium bicarbonate for 90-120 min until capsule was readily visible by India ink stain. Encapsulated bacilli were resuspended in Dulbecco's Modified Eagle's medium containing $10 \%$ heat-inactivated fetal calf serum (DF10) and 10\% type AB human serum (Sigma Aldrich) as a source of complement. Purified human neutrophils were mixed with bacilli in duplicate at an effector : target ratio of $50: 1$ and rotated for $5 \mathrm{~h}$. Bacterial viability was measured by serial dilution in water and plating on LB agar. Results are given as the mean \pm SEM of the duplicate samples. Murine RAW264.7 macrophages were grown and used in an adhesion assay as previously described (Scorpio et al., 2007). Briefly, macrophages were allowed to adhere to circular coverslips overnight or until growth to confluence. Spores were germinated in $\mathrm{BHI} /$ bicarbonate, the bacilli resuspended in DF10 at a concentration of $1 \times 10^{8} \mathrm{ml}^{-1}$ and layered onto the macrophages in a volume of $200 \mu \mathrm{l}$ in duplicate. After $30 \mathrm{~min}$ of incubation at $37^{\circ} \mathrm{C}$, the coverslips were washed extensively with PBS and stained with
Wright-Giemsa. Bacilli adherent to macrophages were counted visually from at least 10 fields of view (at least 100 macrophages) on duplicate coverslips and the mean number of bacilli per macrophage $( \pm$ SEM) determined as previously described (Scorpio et al., 2007).

CapD antibody production. Mice were vaccinated subcutaneously with $10 \mu \mathrm{g}$ recombinant CapD (Scorpio et al., 2007) in $200 \mu \mathrm{l}$ PBS and Ribi R700 adjuvant (Sigma Aldrich), as recommended by the manufacturer. Mice were boosted at 4 weeks and bled from the periorbital sinus at 7 weeks. Blood was collected in serum separator tubes and sera were withdrawn after microcentrifugation for $20 \mathrm{~min}$ at 11000 r.p.m.

Virulence determination. Spores for all challenge experiments were heat-shocked for $40 \mathrm{~min}$ at $65{ }^{\circ} \mathrm{C}$, resuspended in water for injection and administered by intramuscular injection in $200 \mu \mathrm{l}$. Competitive indices were determined by infecting guinea pigs with a mixture containing equal numbers of spores derived from wild-type $B$. anthracis Ames and a capD-overexpressing strain. Guinea pigs were monitored for signs of infection, and when moribund (40-48 h after infection) were euthanized and the spleens removed. The spleens were disrupted by mashing with a $10 \mathrm{ml}$ syringe plunger in a $70 \mu \mathrm{m}$ filter basket placed in a $50 \mathrm{ml}$ centrifuge tube. Spleen cells were washed through the basket with cold PBS. Relative numbers of the wild-type Ames and the capDoverexpressing strain were determined by plating serial dilutions on LB agar containing or lacking antibiotics. Virulence was also determined in guinea pigs by challenge with an intramuscular injection of 2000 spores derived from either wild-type Ames or a capD-overexpressing strain and comparing mean time-to-death.

Statistics. Neutrophil killing and macrophage adherence comparisons were evaluated by analysis of variance (ANOVA) with Tukey's post hoc tests. Differences in virulence were evaluated with Student's $t$-test for competitive index and log rank test for survival curve.

\section{RESULTS}

\section{Translational signalling affects capD expression}

To examine the effect of transcriptional and translational signalling on capD expression, the capD ORF and 
translation initiation site were cloned into pASD2 downstream of the $3^{\prime}$ end of hsp60, EFTu or capA and the resulting plasmids integrated via homologous recombination at the respective genes in B. anthracis Ames. Two transcriptional fusions of capD were made to $h s p 60$, one carrying the wild-type capD RBS and one with a consensus RBS (AGGAGGT). One transcriptional fusion was made to $E F T u$ with a consensus RBS for capD translation. Five transcriptional fusions of capD were made to capA consisting of combinations of an ATG or TTG start codon with the wild-type or consensus RBS. According to the most recent $B$. anthracis genome annotation (Ames ancestor), the stop codon of capA overlaps with the TTG start codon of capD (http://www.ncbi.nlm.gov/nuccore/NC_007323). Construction of the capAcapD fusions carrying a consensus RBS resulted in a $20 \mathrm{bp}$ separation between the capA stop codon and the capD start codon. The capAcapD fusion strains constructed to have TTG or ATG start codons and wild-type RBS had two possible translation start sites separated by $36 \mathrm{bp}$. The capAcapD fusions included capE, which has been shown to be necessary for capsule synthesis (Candela et al., 2005), whereas capE was not included in capD fusions to $h s p 60$ and EFTu, which were made in Ames carrying a functional capE on $\mathrm{pXO}$. Genetic linkages of the capAcapD fusion strains are detailed in Table 3.

Expression of $c a p D$ was examined by immunoblotting of whole-cell lysates from strains grown for $5 \mathrm{~h}$ or overnight $(18 \mathrm{~h})$ at $37^{\circ} \mathrm{C}$ in $\mathrm{BHI} /$ bicarbonate broth. Consistent with our observations that CapD is poorly detected by proteomics analysis, we were unable to detect CapD in lysates of either the parent wild-type Ames strain or a capD mutant at 18 h (Fig. 1a) or 5 h (Fig. 1c). By contrast, CapD was readily detected in lysates of hsp60capDRBS and EFTucapDRBS, in which the predicted capD RBS was replaced with a consensus RBS sequence. CapD was also detected at $5 \mathrm{~h}$ and $18 \mathrm{~h}$ in capAcapDRBSATG, which contains both a consensus RBS and an ATG start codon (Fig. 1a, c). CapD was not detected in capAcapD, in which the pASD2 capAcapDRBSATG plasmid integrated at capD directly after the engineered ATG start codon and formed a wild-type linkage of capD to capA. This strain behaved identically to wild-type Ames in all experiments. Additionally, CapD was not detected in capAcapDRBSTTG, capAcapDTTG or capAcapDATG (Fig. 1a), suggesting that both a consensus RBS and an ATG start codon were necessary for detectable capD expression for capAcapD fusions. Interestingly, for all lysate preparations tested, only the large subunit of CapD reacted with the polyclonal antibody, suggesting that the small subunit was poorly immunogenic, consistent with a previous report (Candela \& Fouet, 2005).

\section{capD expression affects $B$. anthracis phenotype}

The effect of capD overexpression on the phenotype of $B$. anthracis was examined by germinating spores in BHI broth containing $0.8 \%$ bicarbonate under $5 \% \mathrm{CO}_{2}$ and growing them for $5 \mathrm{~h}$ or overnight at $37^{\circ} \mathrm{C}$. Bacilli were visualized by India ink staining and phase-contrast microscopy. When grown in BHI broth, fusions of capD to $h s p 60$ and EFTu in which a consensus RBS was present appeared significantly less encapsulated than the parent wild-type Ames strain, while the capAcapDRBSATG fusion appeared devoid of capsule (Fig. 2). By contrast, capAcapD, capAcapDTTG and capAcapDATG bacilli were encapsulated and appeared similar to wild-type Ames by India ink staining, while capAcapDRBSTTG was slightly less encapsulated (data not shown).

Agarose gel electrophoresis of $\mathrm{BHI} /$ bicarbonate culture supernatants revealed that high-molecular-mass capsule was readily visible in the supernatant from the parent wild-type Ames strain and from capAcapD but was not visible in any of the capD-overexpressing strains, capAcapDRBSATG, EFTucapDRBS and hsp60capDRBS (Fig. 1b). Intermediate-sized capsule was observed in culture supernatants from hsp60capD, while almost entirely low-molecular-mass capsule was observed from capAcapDRBSATG, EFTucapDRBS and hsp60capDRBS. Strain capAcapDTTG appeared to produce capsule of a slightly higher molecular mass than wild-type Ames,

Table 3. Nucleotide linkages of $\operatorname{cap} A c a p D$ fusion strains

RBSs are underlined and the capD start codon and capA stop codon are underlined and in bold.

\begin{tabular}{|c|c|}
\hline Strain & capAcapD linkage \\
\hline Wild-type & TATCAGTGGAGACAACAACTTGAATTCCTTTAAA \\
\hline capAcapDRBSATG & $\begin{array}{l}\text { TATCAGTGGAGACAACAACTTGACTCGAGAGGAGGTCAA } \\
\text { CAACATGAATTCCTTTAAA }\end{array}$ \\
\hline capAcapDRBSTTG & $\begin{array}{l}\text { TATCAGTGGAGACAACAACTTGACTCGAGAGGAGGTCAA } \\
\text { CAACTTGAATTCCTTTAAA }\end{array}$ \\
\hline capAcapDATG & $\begin{array}{l}\text { TATCAGTGGAGACAACAACTTGACTCGAGGAAAATCAAG } \\
\text { TATCAGTGGAGACAACAACATGAATTCCTTTAAA }\end{array}$ \\
\hline capAcapDTTG & $\begin{array}{l}\text { TATCAGTGGAGACAACAACTTGACTCGAGGAAAATCAAG } \\
\text { TATCAGTGGAGACAACAACTTGAATTCCTTTAAA }\end{array}$ \\
\hline
\end{tabular}


(a)

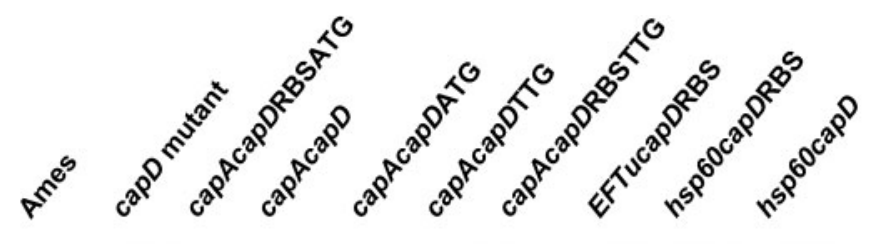

\section{$35 \mathrm{kDa}$}

(b)
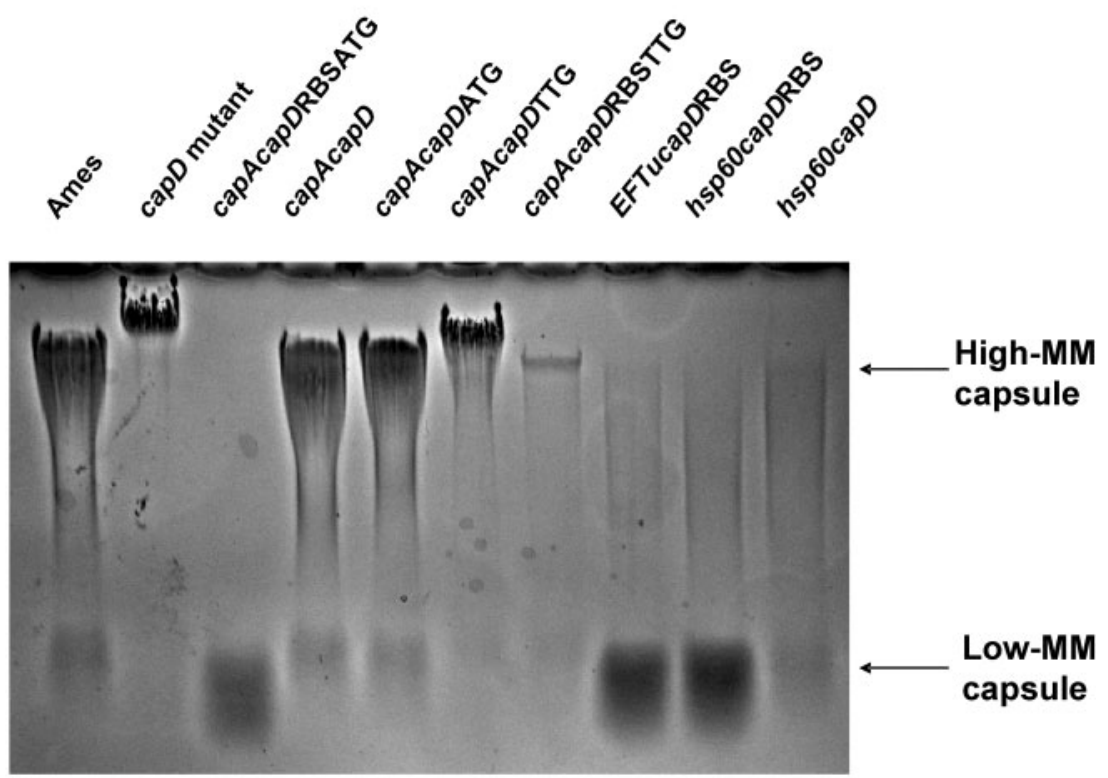

(c)

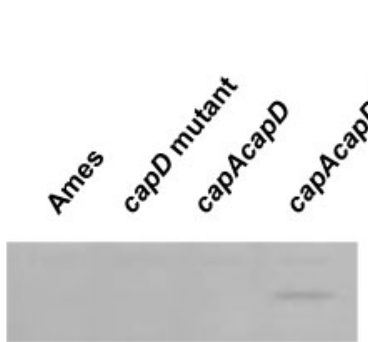

$5 \mathbf{h}$

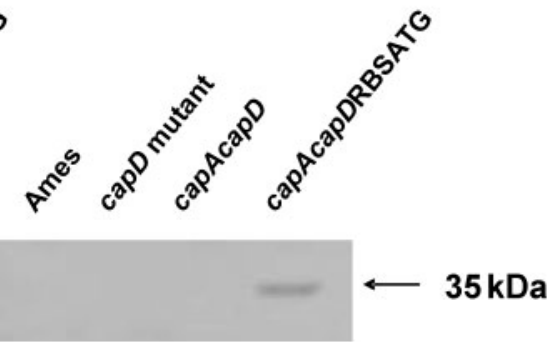

$18 \mathrm{~h}$

Fig. 1. Effect of $c a p D$ linkage on $c a p D$ expression and capsule phenotype. (a) Whole-cell lysates were made for each strain. An equal amount of protein for each sample was separated by electrophoresis in a 4-12\% SDS-Tricine gel and CapD was detected by immunoblotting using a polyclonal mouse anti-CapD serum. (b) Strains were grown in BHI/bicarbonate broth and capsule in the supernatant was examined by agarose gel electrophoresis and staining with methylene blue. Lane identities are identical to (a). (c) Temporal expression of capD determined by immunoblotting of whole-cell lysates 5 or $18 \mathrm{~h}$ after germination.

suggesting a lower level of capD expression, while unexpectedly, capAcapDRBSTTG appeared to produce less total capsule than wild-type Ames (Fig. 1b). The poly-D-glutamic acid nature of the low-molecular-mass capsule was confirmed by reacting it with recombinant CapD and observing degradation on agarose gels (Fig. 3) as described previously for high-molecular-mass capsule (Scorpio et al., 2007). 


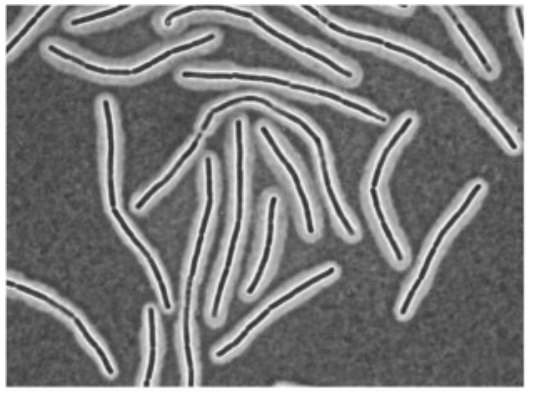

Ames

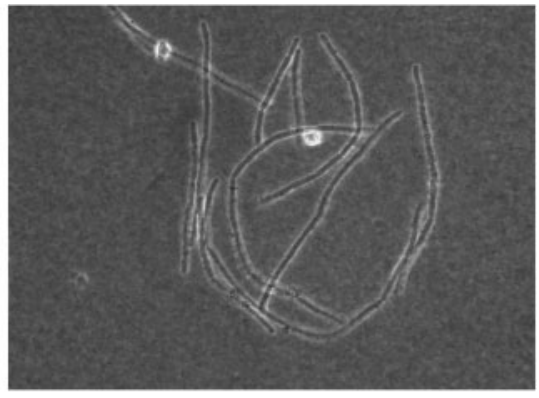

Ames capAcapDRBSATG
Fig. 2. India ink stain of bacilli from $B$. anthracis Ames and capAcapDRBSATG grown overnight in $\mathrm{BHI} /$ bicarbonate and examined by phase microscopy (magnification $\times 700$ ).

\section{Overexpression of capD reduces $B$. anthracis virulence}

The effect of overexpressing capD on $B$. anthracis virulence was examined in cell culture and in a guinea pig infection model. Bacilli from two strains which overexpressed capD, capAcapDRBSATG and EFTucapDRBS, strongly adhered to RAW264.7 murine macrophages, while wild-type Ames failed to adhere (Table 4, $P<0.001$, ANOVA with Tukey's post hoc tests). In addition, bacilli from strains capAcapDRBSATG and EFTucapDRBS were susceptible to neutrophil killing, with $>99 \%$ reduction in viability compared to wild-type Ames bacilli, which were resistant, showing slight growth during the incubation (Table 4, $P<0.001$, ANOVA with Tukey's post hoc tests) as previously reported (Scorpio et al., 2007). Wet-mount microscopy of bacilli and neutrophil mixtures revealed bacilli from capD-overexpressing strains engulfed in phagocytic

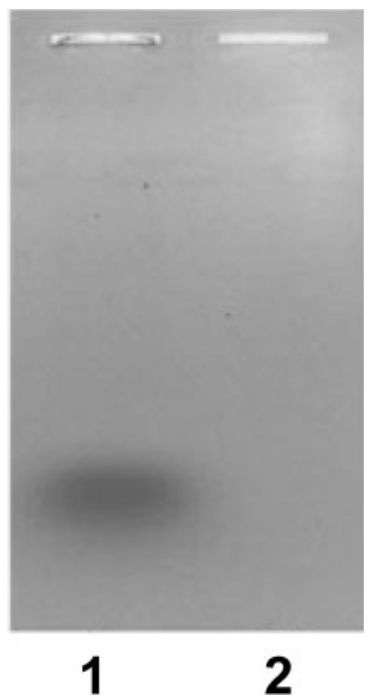

Fig. 3. Low-molecular-mass capsule can be degraded by CapD. EFTucapDRBS supernatant was digested for $2 \mathrm{~h}$ at $37^{\circ} \mathrm{C}$ with $0.3 \mu \mathrm{g} \mathrm{CapD}$ in PBS with $0.1 \mathrm{M}$ serine (lane 2). Undigested capsule was treated in the same way, except that CapD was not included (lane 1). Samples were electrophoresed in $2 \%$ agarose and the gel stained with methylene blue. vacuoles while bacilli from wild-type Ames were almost entirely extracellular (data not shown).

Virulence in guinea pigs was measured by intramuscular injection of wild-type Ames and capAcapDRBSATG spores in a 1:1 ratio (1000 spores of each strain). Animals were euthanized when moribund and the amount of each strain was measured by plating serial dilutions of spleen homogenate onto LB agar with and without antibiotics. Differences in the mean c.f.u. per g spleen between the two strains revealed that capAcapDRBSATG was significantly under-represented relative to wild-type Ames (Fig. 4a, $P=0.011$, Student's $t$-test, $n=4)$. To examine virulence in a non-mixed infection, guinea pigs were infected with 2000 spores of either capAcapD or capAcapDRBSATG and monitored for mortality. Strain capAcapD was chosen for comparison as it contains the pASD2 plasmid integrated at capD but is phenotypically identical to wild-type Ames, suggesting that the integrated plasmid does not have polar effects downstream that may inhibit capsule synthesis. All animals succumbed or were euthanized after infection with both strains. Infection with capAcapDRBSATG resulted in a mean time to death of $54.4 \pm 3.6 \mathrm{~h}(n=5)$ compared with $40.5 \pm 1.0 \mathrm{~h}(n=4)$ for capAcapD, representing a significant increase in survival (Fig. $4 \mathrm{~b}, P=0.0027$, log rank test). By comparison, previous experiments performed with a 2000

Table 4. Macrophage adherence and neutrophil killing of capD-overexpressing strains

Bacilli from the different strains were assayed for macrophage adherence and neutrophil killing as described in Methods.

\begin{tabular}{|lcc|}
\hline Strain & $\begin{array}{c}\text { Macrophage } \\
\text { adherence (no. of } \\
\text { bacilli per cell, } \\
\text { mean } \pm \text { SEM) }\end{array}$ & $\begin{array}{c}\text { Neutrophil killing } \\
\text { (\% viable, } \\
\text { mean } \pm \text { SEM) }\end{array}$ \\
\hline Ames & $0.04 \pm 0.03$ & $181.3 \pm 86$ \\
EFTucapDRBS & $3.9 \pm 1.4^{*}$ & $0.04 \pm 0.01^{*}$ \\
capAcapDRBSATG & $1.9 \pm 0.4^{*}$ & $0.01 \pm 0.001^{*}$ \\
\hline
\end{tabular}

${ }^{\star} P<0.001$ vs wild-type Ames. 
(a)

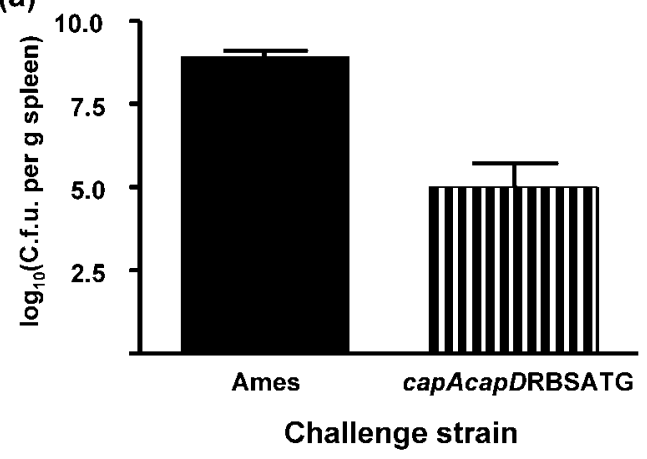

(b)

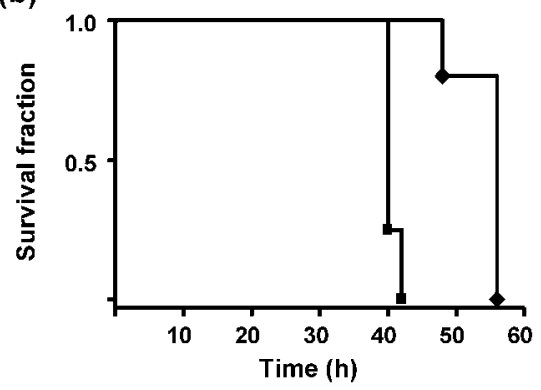

Fig. 4. Effect of $c a p D$ expression on virulence in guinea pigs. (a) Competitive index of capAcapDRBSATG vs Ames. Guinea pigs were challenged with a mixture containing equal numbers of heatshocked spores derived from Ames or capAcapDRBSATG. The number of c.f.u. $\mathrm{g}^{-1}$ from the spleens was counted for each strain by plating on antibiotic-selective medium $(P=0.011)$. Data are presented as log c.f.u. per $g$ spleen. (b) Non-mixed infection. Guinea pigs were infected with 2000 spores of either capAcapD (ם) or capAcapDRBSATG ( $)$ and monitored for mortality. Analysis of Kaplan-Meier survival curves showed that the probability of survival was significantly different $(P=0.0027)$.

spore Ames challenge resulted in a mean time to death of $43.7 \pm 3.3 \mathrm{~h}$ (unpublished observations).

\section{DISCUSSION}

B. anthracis grows to high numbers in the blood and organs of infected animals. This high-density growth is largely a result of resistance to innate immune defence mechanisms conferred in part by the antiphagocytic polyglutamate capsule. CapD has been demonstrated to be a $B$. anthracis virulence factor, contributing to virulence by anchoring the capsule to the peptidoglycan (Candela \& Fouet, 2005) and by releasing low-molecular-mass capsule fragments that may interfere with innate immune function (Makino et al., 2002). We previously showed that exogenous recombinant CapD efficiently degrades the capsule, resulting in increased susceptibility to phagocytic killing. Here we demonstrate that overexpression of capD in $B$. anthracis also results in increased susceptibility to phagocytic killing and decreased virulence in vivo, presumably due to cleavage of capsule from the bacillus surface by excess CapD, exposing the organism to innate immune defence mechanisms.

Despite the fact that capD resides on a single transcript with $c a p B, C$ and $A$, its expression appears to be significantly lower than that of the other genes in the operon (Scorpio et al., 2005). A previous report indicated that CapD was detected by immunoblots of crude extracts from B. anthracis RPG1 (Candela \& Fouet, 2005). However, we were unable to detect CapD in B. anthracis Ames lysates with our polyclonal mouse anti-CapD antibody. The most recent $B$. anthracis genome annotation assigns the capD start codon as part of the capA stop codon, resulting in an overlap of the capD RBS sequence with the $3^{\prime}$ end of capA. Thus, translation of capD may be coupled with capA by a programmed shift of the ORF (Oppenheim \& Yanofsky, 1980) in which the ribosome, upon completion of capA translation, is shifted back along the mRNA to reinitiate translation at the capD RBS sequence. The mechanism that suppresses capD expression may involve a combination of factors. For example, cistron terminal overlap (Shcherbakov \& Garber, 2000) may affect capD translation efficiency due to ribosome competition between capA termination and capD initiation at the overlapping region (Gesteland \& Atkins, 1996). Alternatively, capD expression may be regulated by a translational coupling mechanism in which the mRNA secondary structure inhibits ribosome priming (Rex et al., 1994 ) at the capD translation initiation region. Finally, a weak RBS and TTG start codon may affect capD expression, although TTG start codons are common in Gram-positive bacteria and are reported to have minimal effect on translation efficiency (Ozbudak et al., 2002). We created genetic fusions of $c a p D$ to the $3^{\prime}$ end of the highly expressed genes $h s p 60$ and EFTu and integrated these into the chromosome of $B$. anthracis. Additionally, we created fusions of capD to the $3^{\prime}$ end of capA in which the TTG start codon was replaced with ATG and the putative RBS was replaced with the consensus sequence, AGGAGGT. The recombinant methods used to construct these strains resulted in a $6 \mathrm{bp}$ separation (the XhoI cloning site) between the capA stop codon and the engineered capD RBS compared with the parent Ames strain, in which the capD RBS overlaps with the $3^{\prime}$ end of capA and is separated by $8 \mathrm{bp}$ from the capD start codon. The engineered sequence changes resulted in higher expression of capD as measured by immunoblotting (Fig. 1a). In particular, adding a consensus RBS to capD when fused to $h p s 60$ resulted in detectable CapD compared with no detection observed with the hsp60capD fusion having the wild-type RBS, suggesting that the RBS significantly affects capD translation, similar to the effect of RBS when comparing capAcapDRBSATG with capAcapDATG (Fig. 1a).

Increased capD expression correlated with smaller amounts of surface-associated capsule, presumably due to degradation by CapD. Additionally, the culture supernatant capsule from capD-overexpressing strains in which CapD was detected by immunoblotting was found to be almost 
entirely of low molecular mass. These phenotypic changes were associated with increased phagocytosis of bacilli by human neutrophils and murine macrophages, probably due to reduced surface-associated capsule. Overexpression of $c a p D$ also reduced virulence in guinea pigs as measured by an increase in the mean survival time in a single-strain challenge and reduced fitness in a competitive index experiment comparing the wild-type Ames strain with capAcapDRBSATG. In the single-strain challenge with capAcapDRBSATG, bacilli isolated from the blood and spleen had a capsule that was not detectable by India ink but was visible by FITC-labelled monoclonal anti-capsule antibody stain, suggesting that only minimal surface capsule was required for dissemination from the site of infection for this strain (data not shown). The dissemination and residual virulence of the capAcapDRBSATG strain also supports the hypothesis that CapD-generated lowmolecular-mass capsule contributes to the virulence of B. anthracis (Makino et al., 2002). The higher amounts of low-molecular-mass capsule released from capD-overexpressing strains did not interfere with phagocytosis by neutrophils, as efficient killing of capD-overexpressing strains could be observed even at a 1:1 ratio of neutrophils to bacilli in which $10 \%$ of the assay medium was replaced by supernatant capsule from the capAcapDRBSATG strain (data not shown). This suggests that the capsule associated with the bacillus surface is the primary mechanism for resistance to phagocytosis. It is possible that low-molecular-mass capsule may be important for resistance to other innate immune defence mechanisms such as cationic antimicrobial peptides and complement.

These data demonstrate that overexpression of capD resulted in significant capsule degradation during growth in BHI/bicarbonate broth and probably in vivo, resulting in smaller amounts of surface-associated capsule, an increase in released, low-molecular-mass capsule and reduced virulence. Taken together, the results suggest that fully virulent $B$. anthracis has evolved finely tuned expression of $c a p D$ and that maximum virulence may require both wildtype levels of surface-associated capsule and released lowmolecular-mass capsule.

\section{ACKNOWLEDGEMENTS}

The research described herein was sponsored by the Medical Biological Defense Research Program, US Army Medical Research and Materiel Command, project number A1_X003_04_RD_B. We thank Sarah Norris for her expert statistical analysis. Opinions, interpretations, conclusions and recommendations are those of the authors and are not necessarily endorsed by the US Army. Research was conducted in compliance with the US Animal Welfare Act and other federal statutes and regulations relating to animals and experiments involving animals and adheres to principles stated in the Guide for the Care and Use of Laboratory Animals, National Research Council, 1996. The facility where this research was conducted is fully accredited by the Association for Assessment and Accreditation of Laboratory Animal Care International.

\section{REFERENCES}

Candela, T. \& Fouet, A. (2005). Bacillus anthracis CapD, belonging to the gamma-glutamyltranspeptidase family, is required for the covalent anchoring of capsule to peptidoglycan. Mol Microbiol 57, 717-726.

Candela, T., Mock, M. \& Fouet, A. (2005). CapE, a 47-amino-acid peptide, is necessary for Bacillus anthracis polyglutamate capsule synthesis. J Bacteriol 187, 7765-7772.

Chabot, D. J., Scorpio, A., Tobery, S. A., Little, S. F., Norris, S. L. \& Friedlander, A. M. (2004). Anthrax capsule vaccine protects against experimental infection. Vaccine 23, 43-47.

Day, W. A., Rasmussen, S. L., Carpenter, B. M., Peterson, S. N. \& Friedlander, A. M. (2007). Microarray analysis of transposon insertion mutations in Bacillus anthracis: global identification of genes required for sporulation and germination. J Bacteriol 189, 3296-3301.

Gesteland, R. F. \& Atkins, J. F. (1996). Recoding: dynamic reprogramming of translation. Annu Rev Biochem 65, 741-768.

Green, B. D., Battisti, L., Koehler, T. M., Thorne, C. B. \& Ivins, B. E. (1985). Demonstration of a capsule plasmid in Bacillus anthracis. Infect Immun 49, 291-297.

Kimura, K., Tran, L. S., Uchida, I. \& Itoh, Y. (2004). Characterization of Bacillus subtilis gamma-glutamyltransferase and its involvement in the degradation of capsule poly-gamma-glutamate. Microbiology 150, 4115-4123.

Makino, S., Watarai, M., Cheun, H. I., Shirahata, T. \& Uchida, I. (2002). Effect of the lower molecular capsule released from the cell surface of Bacillus anthracis on the pathogenesis of anthrax. J Infect Dis 186, 227-233.

Menard, R., Sansonetti, P. J. \& Parsot, C. (1993). Nonpolar mutagenesis of the ipa genes defines IpaB, IpaC, and IpaD as effectors of Shigella flexneri entry into epithelial cells. J Bacteriol 175, 5899-5906.

Mendelson, I., Tobery, S., Scorpio, A., Bozue, J., Shafferman, A. \& Friedlander, A. M. (2004). The NheA component of the nonhemolytic enterotoxin of Bacillus cereus is produced by Bacillus anthracis but is not required for virulence. Microb Pathog 37, 149154.

Mock, M. \& Fouet, A. (2001). Anthrax. Annu Rev Microbiol 55, 647-671. Oppenheim, D. S. \& Yanofsky, C. (1980). Translational coupling during expression of the tryptophan operon of Escherichia coli. Genetics 95, 785-795.

Ozbudak, E. M., Thattai, M., Kurtser, I., Grossman, A. D. \& Van, O. A. (2002). Regulation of noise in the expression of a single gene. Nat Genet 31, 69-73.

Rex, G., Surin, B., Besse, G., Schneppe, B. \& McCarthy, J. E. (1994). The mechanism of translational coupling in Escherichia coli. Higher order structure in the atpHA mRNA acts as a conformational switch regulating the access of de novo initiating ribosomes. J Biol Chem 269, 18118-18127.

Richter, S., Anderson, V. J., Garufi, G., Lu, L., Budzik, J. M., Joachimiak, A., He, C., Schneewind, O. \& Missiakas, D. (2009). Capsule anchoring in Bacillus anthracis occurs by a transpeptidation reaction that is inhibited by capsidin. Mol Microbiol 71, 404-420.

Scorpio, A., Wei, J., Ghassemian, M., Su, J., Chen, W., Blank, T. E., Richardson, T. H., Bozue, J. A., Hoover, T. A. \& other authors (2005). The proteome of Bacillus anthracis isolated from infected animals. In Bacillus anthracis, B. cereus, and B. thuringiensis International Conference, p. 20. Santa Fe, New Mexico, USA.

Scorpio, A., Chabot, D. J., Day, W. A., O'Brien, D. K., Vietri, N. J., Itoh, Y., Mohamadzadeh, M. \& Friedlander, A. M. (2007). Poly-gamma- 
glutamate capsule-degrading enzyme treatment enhances phagocytosis and killing of encapsulated Bacillus anthracis. Antimicrob Agents Chemother 51, 215-222.

Scorpio, A., Tobery, S. A., Ribot, W. J. \& Friedlander, A. M. (2008), Treatment of experimental anthrax with recombinant capsule depolymerase. Antimicrob Agents Chemother 52, 1014-1020.

Shcherbakov, D. V. \& Garber, M. B. (2000). Overlapping genes in bacterial and bacteriophage genomes. Mol Biol (Mosk) 34, 572583.
Su, J., Yang, J., Zhao, D., Kawula, T. H., Banas, J. A. \& Zhang, J. R. (2007). Genome-wide identification of Francisella tularensis virulence determinants. Infect Immun 75, 3089-3101.

Uchida, I., Makino, S., Sasakawa, C., Yoshikawa, M., Sugimoto, C. \& Terakado, N. (1993). Identification of a novel gene, dep, associated with depolymerization of the capsular polymer in Bacillus anthracis. Mol Microbiol 9, 487-496.

Edited by: T. J. Mitchell 